\title{
Development of a Taxonomy of Keywords for Engineering Education Research
}

\author{
Cynthia J. Finelli, ${ }^{a}$ Maura Borrego, ${ }^{b}$ and Golnoosh Rasoulifar ${ }^{a}$ \\ ${ }^{a}$ University of Michigan, ${ }^{b}$ The University of Texas at Austin
}

\begin{abstract}
The diversity of engineering education research provides an opportunity for cross-fertilization of ideas and creativity, but it also can result in fragmentation of the field and duplication of effort. One solution is to establish a standardized taxonomy of engineering education terms to map the field and communicate and connect research initiatives. This report describes the process for developing such a taxonomy, the EER Taxonomy. Although the taxonomy focuses on engineering education research in the United States, inclusive efforts have engaged 266 individuals from 149 cities in 30 countries during one multiday workshop, seven conference sessions, and several other virtual and in-person activities. The resulting taxonomy comprises 455 terms arranged in 14 branches and six levels. This taxonomy was found to satisfy four criteria for validity and reliability: (1) keywords assigned to a set of abstracts were reproducible by multiple researchers, (2) the taxonomy comprised terms that could be selected as keywords to fully describe 243 articles in three journals, (3) the keywords for those 243 articles were evenly distributed across the branches of the taxonomy, and (4) the authors of 31 conference papers agreed with $90 \%$ of researcher-assigned keywords. This report also describes guidelines developed to help authors consistently assign keywords for their articles by encouraging them to choose terms from three categories: (1) context/focus/topic, (2) purpose/target/motivation, and (3) research approach.
\end{abstract}

Keywords educational level; educational setting; outcomes; research approaches; theoretical frameworks

\section{Introduction: The Need for a Taxonomy}

Engineering education research is a broad-based, rapidly evolving, diverse, interdisciplinary, and highly international field. Exciting and innovative research is being conducted in a wide variety of educational and professional engineering settings, in countries around the world, and by researchers and practitioners in a range of disciplinary fields (for the range of research, see Alpay \& Jones, 2012; Besterfield-Sacre, Cox, Borrego, Beddoes, \& Zhu, 2014; Borrego, 2007; Borrego \& Bernhard, 2011; Case \& Light, 2011; de Graaff \& Kolmos, 2014; Froyd, Wankat, \& Smith, 2012; Heywood, 2005; Jesiek, Newswander, \& Borrego, 2009). Although this diversity provides an opportunity for cross-fertilization of ideas and creativity, it also can result in fragmentation of the field and duplication of effort.

One solution that would allow engineering education researchers and practitioners to best take advantage of this diversity is to establish a standardized terminology and organizational 
system - a taxonomy of terms - to map the field and to communicate and connect research initiatives. Such a taxonomy of terms for engineering education research could be useful for multiple audiences. It could serve as a framework for researchers and members of the engineering education research community to situate their individual research initiatives in the broader field, see connections and synthesize ideas, better access the research of others, and plan future work. It could be a source of terms to help novices understand the breadth of the field and conduct literature searches. The taxonomy could be a mechanism for journal editors to organize related authors and research areas, identify the alignment of submitted articles with a journal's mission, and categorize a reviewer database for more appropriate assignment of reviewers. And it could provide a method for funding agencies to classify research portfolios, identify areas for capacity building, frame solicitations, and recruit reviewers.

Others have noted the importance of organizing research areas and publications in the field of engineering education research and have established systems for categorizing the field and analyzing entire bodies of work. Wankat $(1999,2004)$ and Whitin and Sheppard (2004) are frequently credited with the earliest efforts in analyzing the field; each catalogued a set of articles published in the Journal of Engineering Education. Among other things, they studied each article's research topic, theoretical framework, and approaches to data collection. More recently, Jesiek et al. (2011) studied over 2,000 publications in a variety of global engineering education journals and conferences over a three-year period, extracting 38 categories of terms; Malmi and collaborators (e.g., 2012, 2013) have categorized papers from the Société Européenne pour la Formation des Ingénieurs (European Society for Engineering Education [SEFI]) annual conferences, and focused particularly on the research process; and Xian and Madhavan (2014) analyzed over 24,000 papers from engineering education conferences and journals and used the taxonomy of education topics in the Education Resources Information Center (ERIC) database to characterize networks of collaboration among scholars.

Although different approaches such as these help identify a list of terms that might map the field of engineering education research, a standardized taxonomy would be more useful. It would guide researchers, journal editors, funding agencies, and other members of the community in creating the metadata that would enable deeper and more extensive analysis of research and publication trends.

In this report, we describe our process for creating a useful taxonomy and establishing an inclusive process for doing so, and we offer evidence that the taxonomy is valid and reliable. Although this taxonomy does focus on engineering education research in the United States, we describe how we engaged a broad, international set of stakeholders in developing the taxonomy, and we hope the report informs and complements related efforts in the international community.

We also describe guidelines we developed for assigning keywords, and we hope those guidelines offer a useful framework for describing an author's work. The guidelines are not intended to constrain authors to using terms from the taxonomy for titles, abstracts, and the body of their manuscripts, and we expect journals, funders, and other organizations to set their own policies for using the taxonomy. Benefits of using standardized terms as we suggest include the ability to both connect related work and help others find engineering education research publications, and these benefits extend beyond the United States. And consistent with accepted taxonomy management practices, we propose that our field establish a committee of representatives from the journals jointly publishing this report to regularly maintain and update the taxonomy so it remains relevant and useful. 


\section{Basic Taxonomy Definitions and Concepts}

A taxonomy, a concept borrowed from biology, is a hierarchical classification scheme that makes categorizing or finding terms within bodies of text easier for both indexers and searchers (Pack, 2002). A taxonomy typically consists of a controlled vocabulary, or restricted list of terms for indexing, a set of preferred terms with the ways similar or related terms might be crossreferenced, and a hierarchy, which describes relationships among the vocabulary terms (Hedden, 2010). A taxonomy, specifically the preferred terms, can help researchers search the literature by linking and suggesting related terms and by offering a hierarchical structure that aids in navigation (Hedden, 2010). Most engineering databases, including IEEE, INSPEC, and Compendex, use controlled vocabularies, which assist users in locating related publications, sometimes without the users' conscious knowledge of the controlled vocabulary's existence. In other fields, some publications rely on databases that use controlled vocabularies (e.g., ERIC and IEEE Xplore) to assign keywords (Hedden, 2010), but there is no such consistency in the field of engineering education research. A good taxonomy has uniform depth (level of detail), comprises distinct concepts represented by one preferred term, and uses the native language of the community it serves (Pack, 2002).

Policy guidelines for applying a taxonomy can help researchers follow consistent procedures by establishing the proper level of detail for keywords, providing a framework of aspects to consider when selecting terms for a document, and specifying how many terms to apply. Taxonomy developers typically generate terms by consulting subject matter experts and studying the corpus of literature in which a taxonomy will be applied (Hedden, 2010). Then, a taxonomy should evolve through regular use - it should be flexible, easily updated, and constantly maintained (Levy, 2004). Users who apply a taxonomy should be able to offer suggestions for adding new terms, removing old terms, clarifying existing terms, and making other updates. Similarly, individuals who maintain a taxonomy should monitor terms for overuse (which indicates the need to split one term into multiple terms), underuse (which suggests the need to delete or combine terms), and misuse (which highlights the need to clarify terms) (Hedden, 2010). Taxonomies developed for academic fields can also be updated through a concerted effort of the broader community on a regular cycle, such as every five years (Marjorie Hlava, personal communication).

\section{Taxonomy Uses}

A taxonomy can be used in many ways. For instance, it can serve as a source of keywords to describe a field of research, and it can provide a more precise approach for finding related documents than full-text searching does. As Levy (2004) noted, when using an indexed taxonomy as the framework for searching,

It's easy to get interesting synergies and to find items using new terms and jargon. A taxonomy is more reliable and standard, and it expresses the information architecture, providing context and grouping even when the words used are different. It makes search results more accurate and understandable, provides alternate access, uses different vocabulary, and crosses boundaries. (p. 11)

Further, taxonomies and controlled vocabularies can help users focus on important sources from among literally millions of results, as noted by Hawkins, Larson, \& Caton (2003): 
When databases of information (particularly in full text) first became available on the Internet, many users felt that thesauri and subject classifications were no longer needed and would go the way of horseless carriages. After all, the theory ran, if everything is available on-line in full text, one would only need to enter the appropriate terms into a search engine, and the desired information would be retrieved. Inexperienced searchers quickly discovered the fallacy of this approach to information retrieval when they were faced with result sets numbering in the millions of hits, with the desired information buried somewhere in them. It was soon recognized that, far from fading away in the Web environment, subject classifications and thesauri have become more important than ever, and organizing information into subjects, or taxonomies, provided users with a significant improvement in retrieval. (p. 772)

A taxonomy can also offer a structure to connect related research and enable visualization of the relationships between publications or other sources to which is it applied (Pack, 2002). For example, products such as SciVal and Pivot use taxonomies and publication data from Scopus and ProQuest databases (respectively) to identify funding opportunities and potential collaborators. Elsevier also offers Reviewer Finder to find reviewers and expand a reviewer network, and the Deep Insights Anytime, Anywhere project creates user-friendly interfaces for searching and visualizing the database of grant projects and investigators of the Transforming Undergraduate Education in STEM program of the United States National Science Foundation (Liu, Vorvoreanu, Madhavan, \& McKenna, 2013).

After the initial dataset is hand-coded and decisions about terminology and relevant category names are made by humans, a taxonomy can serve as the basis for automated algorithms that organize content (including web content) to find and categorize information (Pack, 2002). In engineering education, for instance, Xian and Madhavan (2014) created a machineassigned method for analyzing the topics of engineering education publications using the human-generated taxonomy of education topics in the ERIC database.

\section{Taxonomies in Other Fields}

Other fields have applied taxonomies for better defining their scope and boundaries, assigning reviewers, and organizing biological data. For example, Ulrich and Dash (2011) developed a taxonomy for the Journal of Research Practice to strengthen the profile and visibility of its transdisciplinary content, to communicate more precisely to potential authors about the "fit" of their submission within the journal's scope, and to allow potential readers to judge their interest in the journal (and to recommend it to others). Their taxonomy is organized using three distinct categories: (1) focus areas (broad theme of importance to the journal), (2) subject areas, and (3) keywords. Ulrich and Dash (2011) noted,

Basically, we believe that defining focus areas is a useful way to clarify and explain to our readers and authors those topics of particular interest in which the journal aims to excel, that is, to achieve a high standard of quality, originality, and practical relevance. (p. 3)

In the field of computer science and information systems, Kalmukov (2012) described how automated computer algorithms could use a taxonomy of terms and information from individual editors or program chairs to assign peer reviewers with overlapping but nonidentical keyword profiles. He conducted an experiment in which conference papers were assigned a set of keywords, potential reviewers self-identified their expertise, and reviewers then bid on 
willingness to review specific papers according to the perceived match with their expertise. The experiment, though, resulted in poor matches between the automated assignments, and Kalmukov identified several reasons for these mismatches. He found that reviewers did not select enough keywords to cover their entire range of reviewing competency areas, reviewers often used keywords that were too general to describe the specific papers, the taxonomy had duplicate terms in multiple areas, and many papers lacked sufficient keywords to describe their complete interdisciplinary scope.

The Gene Ontology Consortium is a virtual community of volunteer editors who since 1996 have maintained structured, controlled vocabularies of gene product function information (Mayor \& Robinson, 2014). The ontology provides additional uniformity to the way that biologists apply the conventions of naming and describing the functions of genes. Although the ontology does not focus on keywords that would be assigned to publications or authors, its effectiveness can still be tracked through bibliometric analysis of publications. Mayor and Robinson (2014) found that publications using the ontology are numerous, increasing in number, and represent a global research community. That the papers using the ontology are published in a wide range of journals and are cited frequently attest to the usefulness of the ontology. These results suggest a vision for widespread use of the engineering education research taxonomy described here.

\section{Evolution of the Taxonomy: A Design-Based Research Approach}

To meet the need for a taxonomy in engineering education research, we applied the integrative learning design framework from design-based research in education (Kelly, 2014). Specifically, we developed an initial draft taxonomy and then simultaneously refined and tested draft versions of the taxonomy by engaging various stakeholders through multiple iterations of deployment and improvement, resulting in the final taxonomy, EER Taxonomy Version 1.0 (Finelli \& Borrego, 2015). We evaluated the validity and reliability of the taxonomy and developed guidelines for using it. For our work, we had two specific goals:

Create a useful taxonomy We aimed to create a concise and comprehensive map for the field that would be complete enough to allow any research initiative to be described using four to six keywords but that would not be an exhaustive list of every possible term. We also intended for the taxonomy to be a useful tool for multiple audiences, including researchers and other community members in the field, journal editors, and funding agencies. We worked to achieve this goal through a process of continuous improvement by seeking feedback and advice from an advisory board of journal editors and other recognized experts in engineering education, conducting several iterative refinements of the taxonomy, hearing from multiple and varied voices at all stages of the process, and seeking out a professional taxonomist early in the project with whom we partnered through the entire process.

Establish an inclusive process Although this taxonomy project was funded by the U.S. National Science Foundation, we employed a process that aimed to be inclusive of the diverse global community of engineering education research. Thus, we included international participation of faculty, administrators, and graduate students from across the world through our advisory board, invited workshops, and presentations at international conferences. Many of our design decisions were guided by this purpose of 
inclusiveness. We advertised opportunities for involvement broadly through national and international venues. When space for participation was limited, we used transparent selection processes and criteria that encouraged diversity. At workshops and conference sessions, we implemented activities that de-emphasized hierarchy and privilege of particular research areas, and we adapted activities to be relevant in the local context for engineering education research. Ultimately however, due to time and funding constraints, the taxonomy focuses on engineering education research in the United States.

Our approach included proactive and retroactive analysis of data and other feedback from multiple members of the community, collected through one workshop, seven conference sessions, and other activities. Table 1 provides details about those individuals, and Figure 1 maps their home institutions. Altogether, there were 266 unique participants, representing 149 cities in 30 countries, who engaged in a series of activities that involved: (1) refining draft versions of the taxonomy, (2) evaluating validity and reliability of the taxonomy, and (3) developing a set of guidelines for assigning keywords from the taxonomy. Each step is described in detail below.

Table 1 Project Activities, Conference

Details, and Participant Demographics

\begin{tabular}{|c|c|c|c|}
\hline & \multicolumn{3}{|c|}{ Participants } \\
\hline & U.S. & Non-U.S. & Total \\
\hline \multicolumn{4}{|l|}{ Refining the taxonomy } \\
\hline Advisory board meetings, 2013-2014 & 7 & 2 & 9 \\
\hline Commissioned outlines, April 2013 & 3 & 3 & 6 \\
\hline $\begin{array}{l}\text { Mapping the Field, May 20-21, } 2013 \\
\text { Ann Arbor, Michigan, USA }\end{array}$ & 39 & 9 & 48 \\
\hline $\begin{array}{l}2013 \text { ASEE, June 25, } 2013 \\
\text { Atlanta, Georgia, USA }\end{array}$ & 35 & 6 & 41 \\
\hline $\begin{array}{l}2013 \text { REES, July 6, } 2013 \\
\text { Kuala Lumpur, Malaysia }\end{array}$ & 12 & 48 & 60 \\
\hline Virtual workshop for graduate students, Aug. 2013 & 8 & 1 & 9 \\
\hline $\begin{array}{l}2013 \text { SEFI, Sept. 20, } 2013 \\
\text { Leuven, Belgium }\end{array}$ & 3 & 54 & 57 \\
\hline $\begin{array}{l}2013 \text { FIE, Oct. 25, } 2013 \\
\text { Oklahoma City, Oklahoma, USA }\end{array}$ & 29 & 7 & 36 \\
\hline $\begin{array}{l}\text { Evaluating validity and reliability } \\
2014 \text { ASEE, June } 2014 \\
\text { Indianapolis, Indiana, USA }\end{array}$ & 27 & 3 & 30 \\
\hline $\begin{array}{l}\text { Developing guidelines for assigning keywords } \\
2014 \text { FIE, Oct. 22, } 2014 \\
\text { Madrid, Spain }\end{array}$ & 6 & 3 & 9 \\
\hline Total unique participants ${ }^{\mathrm{a}}$ & 137 & 129 & 266 \\
\hline
\end{tabular}

a Some individuals participated in more than one activity, so the number of unique participants is not a summation of the overall participant count. 


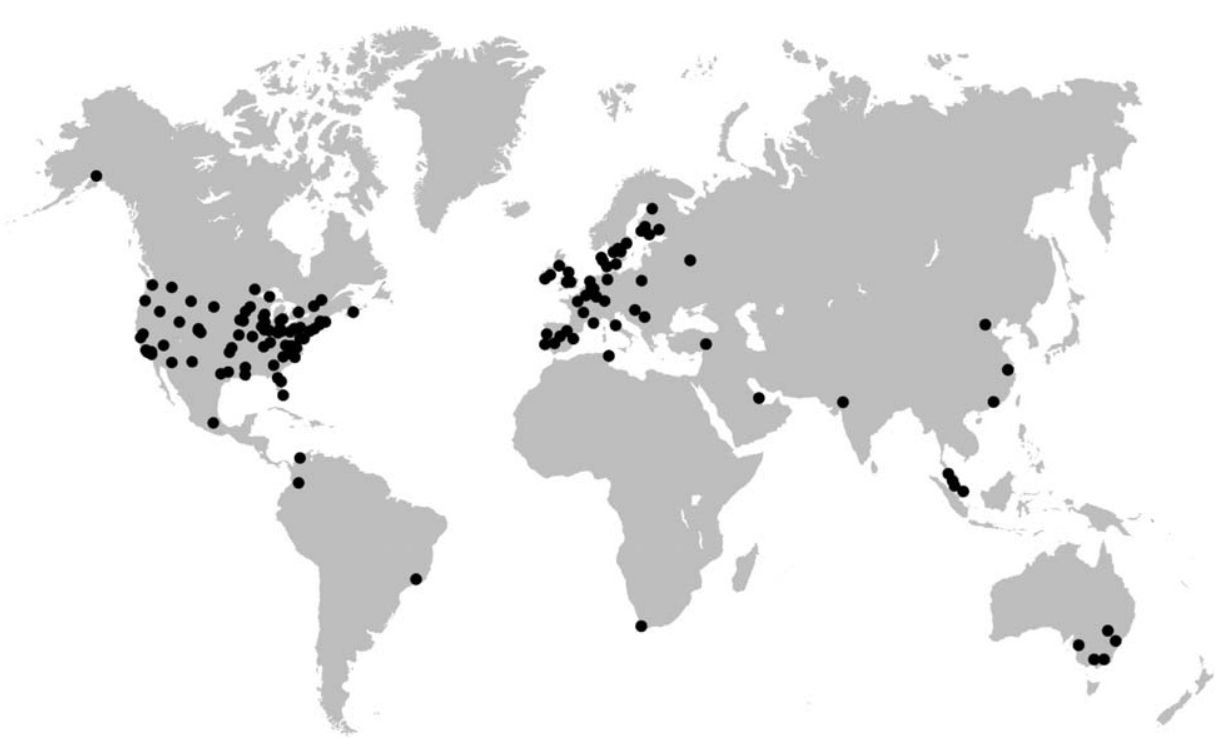

Figure 1 Location of home institution for 266 unique participants.

\section{Refining the Taxonomy*}

The diverse range of community members who assisted in developing and refining our taxonomy included experts from Access Innovations, an information management firm that regularly creates and maintains taxonomies. We developed an initial draft taxonomy from three commissioned outlines, participants refined the draft prototype at a two-day Mapping the Field Conference, and stakeholders developed and refined draft versions at a series of five subsequent workshops: the 2013 American Society for Engineering Education Annual Conference, the 2013 Research in Engineering Education Symposium, a virtual workshop that engaged nine graduate students, the 2013 European Society for Engineering Education Annual Conference, and the 2013 Frontiers in Education Annual Conference. At each iteration, we allowed stakeholders to practice on a diverse set of articles (Hedden, 2010), and we followed ANSI/NISO Z39.19 standard in carefully considering which distinct terms to include and which compound words warranted a unique term (Hedden, 2010). Details about the six draft versions of the taxonomy (versions 0.1 through 0.6 ), as well as the final taxonomy (EER Taxonomy Version 1.0), are included in Table 2.

Advisory board meetings To guide our project efforts, we established a 10-member advisory board comprising leaders in engineering education. The board included the chairs of two departments of engineering education (Purdue University and Virginia Polytechnic Institute and State University), the past or present editors of five journals in the field (Australasian Journal of Engineering Education, European Journal of Engineering Education, Journal of

\footnotetext{
*The majority of text in this section is reprinted with permission, (C) 2014 American Society for Engineering Education (Finelli \& Borrego, 2014). This report differs from the conference papers cited within it, because it includes a complete description of all sessions as well as an expanded literature review and comprehensive analysis in the discussion and conclusion.
} 
Table 2 Details of Taxonomy Versions

\begin{tabular}{lcrcc}
\hline & Date created & $\begin{array}{r}\text { No. of } \\
\text { terms }\end{array}$ & $\begin{array}{c}\text { No. of } \\
\text { branches }\end{array}$ & $\begin{array}{c}\text { No. of } \\
\text { levels }\end{array}$ \\
\hline Version 0.1 & $05 / 05 / 13$ & 1,257 & 13 & 7 \\
Version 0.2 & $06 / 21 / 13$ & 969 & 14 & 6 \\
Version 0.3 & $07 / 01 / 13$ & 1,012 & 14 & 6 \\
Version 0.4 & $09 / 13 / 13$ & 1,057 & 17 & 6 \\
Version 0.5 & $10 / 17 / 13$ & 1,052 & 17 & 6 \\
Version 0.6 & $11 / 08 / 13$ & 452 & 14 & 6 \\
Version 1.0 & $09 / 23 / 14$ & 455 & 14 & 6 \\
\hline
\end{tabular}

aThe branch names are included in the counts for "number of terms."

Engineering Education, IEEE Transactions on Education, and International Journal of Engineering Education), and other recognized world leaders in engineering education research. Members of the advisory board are listed in Table 3.

We consulted with our advisory board at two in-person meetings (one immediately following the Mapping the Field Conference in Ann Arbor and one during the 2013 American Society of Engineering Education Annual Conference), at multiple virtual meetings, and through individual phone conversations. At each phase, we reviewed the process for engaging the community in refining the taxonomy, considered whether the goals and focus (with respect to research vs. practice and national vs. international) were appropriate and achievable, and discussed strategies for ensuring access and use of the final taxonomy. The advisory board was particularly helpful in thinking strategically about the long-term impact of the project.

Commissioned outlines Participant selection We began the project by commissioning three outlines to synthesize related efforts already underway. We issued an international, open call and received six proposals for initial outlines. From the proposals submitted, we commissioned three teams (six individuals) to provide outlines as the basis for the initial taxonomy.

Outline details The three outlines, each consisting of terms arranged in a two-level outline, were developed using different approaches and source materials. For the first outline, its authors began by analyzing bibliographic data (i.e., title, abstract, and keyword list) for nearly 900 international journal articles and conference papers from 2005 to 2008. They iteratively created a set of first-level terms (each having a clearly identifiable research area) and secondlevel terms. Next, from over 500 relevant journal articles and conference proceedings, the authors expanded their analysis by creating a more comprehensive set of terms for the diversity category. Their resulting outline contained 1,242 terms categorized into 38 branches.

The second outline was based on 2,216 peer-reviewed journal articles in five international engineering education journals from 1959 to 2012. The authors of the outline studied the titles to determine an initial set of keywords for each article, and then they applied network analysis to identify underlying themes and relationships among the keywords. The two-level outline included 256 terms categorized into 46 branches, as well as a time dimension and an indication of the level of connectedness. 
Table 3 Advisory Board Members

\begin{tabular}{|c|c|}
\hline Stephanie Adams & $\begin{array}{l}\text { Department Head and Professor, Engineering Education } \\
\text { Virginia Polytechnic Institute and State University, Blacksbu }\end{array}$ \\
\hline Cindy Atman & $\begin{array}{l}\text { Director, Center for Engineering Learning \& Teaching and } \\
\text { and Lella Blanche Bowie Endowed Chair, Human Centered } \\
\text { University of Washington, Seattle, Washington, USA }\end{array}$ \\
\hline Erik de Graaff & $\begin{array}{l}\text { Editor-in-Chief, European Journal of Engineering Education } \\
\text { and } \\
\text { Professor, Aalborg University, Aalborg, Denmark }\end{array}$ \\
\hline Jeffrey Froyd & $\begin{array}{l}\text { Editor-in-Chief, IEEE Transactions on Education } \\
\quad \text { and } \\
\text { Director, Faculty Climate and Development and TEES Res } \\
\text { Texas A\&M University, College Station, Texas, USA }\end{array}$ \\
\hline Ahmad Ibrahim & $\begin{array}{l}\text { Editor, International Journal of Engineering Education } \\
\quad \text { and } \\
\text { Professor, RCC Institute of Technology } \\
\text { Yorkville University, Toronto, Ontario, Canada }\end{array}$ \\
\hline Euan Lindsay & $\begin{array}{l}\text { Past co-editor, Australasian Journal of Engineering Education } \\
\text { and } \\
\text { Foundation Professor of Engineering } \\
\text { Charles Sturt University, Bathurst, New South Wales, Austr }\end{array}$ \\
\hline Thomas Litzinger & $\begin{array}{l}\text { Director, Leonhard Center and Professor, Mechanical Engir } \\
\text { The Pennsylvania State University, University Park, Pennsyl }\end{array}$ \\
\hline David Radcliffe & $\begin{array}{l}\text { Kamyar Haghighi Head and Epistemology Professor, Engin } \\
\text { Purdue University, West Lafayette, Indiana, USA }\end{array}$ \\
\hline Michael Loui & $\begin{array}{l}\text { Editor, Journal of Engineering Education } \\
\quad \text { and } \\
\text { Dale and Suzi Gallagher Professor, Engineering Education } \\
\text { Purdue University, West Lafayette, Indiana, USA } \\
\quad \text { and } \\
\text { Professor Emeritus, Electrical and Computer Engineering } \\
\text { University of Illinois at Urbana-Champaign, Illinois, USA }\end{array}$ \\
\hline
\end{tabular}

Sheri Sheppard Co-Director, Center for Design Research and Professor, Mechanical Engineering Stanford University, Stanford, California, USA

The third outline involved analyses of three separate sources: (1) the full text of 35 journal articles published in the United Kingdom during 2006-2012, 86 conference papers during 2008-2012, and a pedagogic research statement for the field; (2) the authors' personal reflections on three related conferences and a study of relevant Ph.D. theses; and (3) meeting notes of the United Kingdom Engineering Education Research Special Interest Group from 2009 to 2013. The two-level outline included 56 terms categorized into 13 branches.

Outcome From the three commissioned outlines, Access Innovations created a draft taxonomy. The company integrated and refined the outlines, normalized the terms, and worked to consolidate the two-level structure into a more hierarchical one. The resulting draft taxonomy (version 0.1) included 1,257 terms arranged in 13 branches and seven levels.

Mapping the Field Conference Participant selection The Mapping the Field Conference was a two-day workshop to refine the draft taxonomy and its accompanying organizing 
structure. We sent email invitations to several groups, including the Research in Engineering Education Network, the Global Engineering Deans Council of the International Federation of Engineering Education Societies, the Educational Research and Methods Division of the American Society of Engineering Education, and the Engineering Libraries Division of the American Society of Engineering Education. The invitation included a link to a brief application, which requested individuals to list keywords to describe their main research area and to answer two questions: What areas of engineering education are in most pressing need of additional research? How will you contribute to diversity of perspectives at this workshop?

We aimed to be as inclusive of the international community as possible, and we selected a group of researchers and other stakeholders according to a variety of factors, including diversity of experience and history in the field; diversity of research area and understanding of the field based on application responses; and diversity of perspectives, especially as related to international backgrounds. Altogether, we selected 48 conference participants from 96 applicants. The participants comprised 39 individuals from the United States and nine colleagues from other countries and who represented 40 distinct institutions from across the world. They had broad experience levels and ranged from current graduate students and faculty new to the field, to seasoned veterans with extensive experience.

Conference details The Mapping the Field Conference was held in Ann Arbor, Michigan, on May 20-21, 2013. Participants reviewed subsections of the initial draft taxonomy in advance of the conference and worked to further refine version 0.1 of the draft taxonomy. At the conference, a professional taxonomist from Access Innovations presented an introduction to taxonomies and an overview about using and maintaining taxonomies. Participants engaged in multiple breakout sessions where they discussed subsections of the taxonomy, suggested term-by-term revisions, and made high-level revisions to the overall taxonomy.

Outcome Access Innovations worked with the documents produced at the conference, and through an iterative process, we produced version 0.2 of the draft taxonomy. It included 969 total terms, arranged in 14 branches and six levels.

2013 American Society of Engineering Education Annual Conference Workshop details We held a free workshop at the 2013 American Society of Engineering Education (ASEE) Annual Conference in Atlanta, Georgia, on June 25, 2013 (Finelli \& Borrego, 2013). This pre-conference workshop was advertised to all conference attendees by means of an abstract printed in the conference program. The abstract included specific welcoming statements to indicate the inclusive nature of the workshop. During the three-hour workshop, 41 participants reviewed version 0.2 of the draft taxonomy and used and revised it during two activities.

For the first activity, we compiled six sets of five abstracts, selected from papers published in the Proceedings of the 2012 ASEE Annual Conference and in recent issues of Advances in Engineering Education, the International Journal of Engineering Education, and the Journal of Engineering Education. Working individually, then in small groups, participants applied the draft taxonomy to abstracts in one of the sets by identifying four to six keywords to describe each abstract. They also identified terms missing from the taxonomy and offered suggestions for making the taxonomy easier to use. Then, for the second activity, participants assisted in refining the taxonomy by working in groups to make detailed, term-by-term revisions to selected branches of the draft taxonomy.

Outcome Following the 2013 ASEE Annual Conference, we integrated participant feedback to produce version 0.3 of the draft taxonomy. It included 1,012 total terms, arranged in 14 branches and six levels. 
2013 Research in Engineering Education Symposium Workshop details To solicit insights representing a global set of perspectives about the taxonomy, we held a free workshop at the 2013 Research in Engineering Education (REE) Symposium in Kuala Lumpur, Malaysia, on July 6, 2013. We advertised the workshop to all symposium attendees through an inclusively worded abstract printed in the conference program, and 59 participants reviewed version 0.3 of the draft taxonomy during a 90-minute post-conference session. As at the previous conference, participants began by using the draft taxonomy to identify keywords for a set of abstracts. To be relevant to the context of the symposium, the activity involved five sets of four abstracts, chosen from papers published in the Proceedings of the 2011 REE Symposium, in the Proceedings of the 2012 REE Symposium, and in recent issues of the International Journal of Engineering Education. Participants then engaged in a broader discussion about making the taxonomy more globally relevant and ensuring its widespread use.

Outcome Several suggestions for refining the taxonomy resulted from the workshop activities. Rather than create an updated taxonomy, we used these suggestions to inform a virtual workshop for graduate students.

Virtual workshop for graduate students Participant selection To further refine the taxonomy over a longer period of time than a conference workshop, we invited graduate students to apply to participate in a virtual workshop through a series of broadly distributed email messages. We asked applicants to complete a keyword activity and respond to questions about transformative research in engineering education and about how they would contribute to diversity in the group. Drawing on their responses, we selected nine students to participate.

Workshop details Over a one-month period, the graduate students reviewed version 0.3 of the draft taxonomy and completed three activities. They worked individually to use and apply the taxonomy to identify keywords for five abstracts of their choosing, participated in virtual group work (in teams of three) to revise an assigned portion of the daft taxonomy using feedback generated from the 2013 REE Symposium, and worked in virtual groups to make holistic revisions to a new version of the taxonomy.

Outcome We incorporated feedback from these activities to develop version 0.4 of the draft taxonomy. It included 1,057 total terms, arranged in 17 branches and six levels.

2013 European Society for Engineering Education Annual Conference Workshop details To again collect insights representing a broad, international perspective on the taxonomy, we held a workshop at the 2013 European Society for Engineering Education (SEFI) Annual Conference in Leuven, Belgium, on September 20, 2013 (Borrego, 2013). This workshop was advertised to all conference attendees, and the SEFI Engineering Education Research Working Group members were especially encouraged to attend. Fifty-seven participants reviewed version 0.4 of the draft taxonomy during the four-hour post-conference workshop, and they engaged in one activity to use the taxonomy and a second activity to refine it. Abstracts for the applying the taxonomy activity were chosen from papers published in the Proceedings of the 2011 SEFI Annual Conference, in the Proceedings of the 2012 SEFI Annual Conference, and in the 2013 volume of the European Journal of Engineering Education. Participants also offered suggestions for higher-level revisions to the taxonomy.

Outcome Following the 2013 SEFI Annual Conference, we integrated participant feedback and produced version 0.5 of the draft taxonomy. It included 1,052 terms, arranged in 17 branches and six levels.

2013 Frontiers in Education Annual Conference Workshop details We conducted a free workshop at the 2013 Frontiers in Education (FIE) Annual Conference in Oklahoma City, Oklahoma, on October 23, 2013 (Finelli, 2013). The workshop was advertised to all conference 
attendees through the conference program. During the three-hour, pre-conference session, 35 participants reviewed version 0.5 of the draft taxonomy and engaged in two activities. The first activity involved using the taxonomy to identify keywords for four abstracts selected from papers published in the Proceedings of the 2011 FIE Annual Conference and in the Proceedings of the 2012 FIE Annual Conference. In a second activity, smaller groups revised one of six branches of the taxonomy.

Outcome Following the 2013 FIE Annual Conference, we integrated feedback from participants to develop version 0.6 of the draft taxonomy. It was a significantly streamlined version, which included just 452 terms organized in 14 branches and six levels. As suggested by workshop participants, these changes were made for two reasons: to achieve a consistent level of detail across all of the taxonomy branches, and to focus the taxonomy more on existing work than aspirational or future directions.

In summary, we conducted several in-person and virtual workshops, integrated feedback from our advisory board, and worked with a taxonomy creation firm to develop and refine draft versions of the taxonomy. Our use of inclusive activities to solicit a broad range of global perspectives produced a draft taxonomy that we assessed for validity and reliability.

\section{Evaluating Validity and Reliability}

To evaluate the validity and reliability of the taxonomy, we adapted the procedures Hawkins et al. (2003) used to validate their information science taxonomy. In this procedure, multiple researchers assigned keywords to a set of publication abstracts, studying the resulting keywords, and (because they did not involve their community in many iterations of developing the taxonomy) refining the taxonomy by adding some terms and removing others that were unused or duplicated. Since we extensively involved our community in refining the taxonomy, we went beyond these efforts by adding a procedure, related to reliability, which assessed the extent to which authors agreed with keywords we assigned to describe their publications. Overall, through a series of activities involving conference papers and journal articles, we evaluated the validity and reliability of our taxonomy using four criteria:

1. Keywords assigned from the taxonomy are reproducible by different researchers.

2. The field of engineering education research, as represented by a sample of journal articles, is encompassed by terms in the taxonomy.

3. Keywords assigned from the taxonomy to the same journal articles are evenly distributed across the branches.

4. Keywords assigned from the taxonomy to a set of conference papers representative of the field are reliable.

Norming exercise Activity details We completed a norming exercise to provide a consistent, objective approach for selecting keywords and to identify the extent to which keywords were reproducible by multiple researchers. After developing a set of working guidelines for assigning keywords, two of us independently assigned keywords from version 0.6 of the draft taxonomy to the same 30 abstracts used at the 2013 ASEE Annual Conference workshop. Then we compared the keywords. We also refined the working guidelines for assigning keywords to ensure even more consistency for subsequent activities. 
Table 4 Papers Published and Abstracts

Reviewed for Evaluating Validity and Reliability

\begin{tabular}{|c|c|c|c|c|c|c|c|c|}
\hline & \multicolumn{4}{|c|}{ No. papers published } & \multicolumn{4}{|c|}{ No. abstracts reviewed } \\
\hline & 2011 & 2012 & 2013 & Total & 2011 & 2012 & 2013 & Total \\
\hline Journal of Engineering Education & 30 & 29 & 22 & 81 & 30 & 29 & 22 & 81 \\
\hline IEEE Transactions on Education & 82 & 73 & 60 & 215 & 30 & 29 & 22 & 81 \\
\hline International Journal of Engineering Education & 130 & 112 & 142 & 374 & 30 & 29 & 22 & 81 \\
\hline Total & 242 & 214 & 224 & 680 & 90 & 87 & 66 & 243 \\
\hline
\end{tabular}

Outcome We found extensive overlap between the keywords assigned by the two of us. There were a few disagreements, but those were easy to resolve. Because there was a high level of reproducibility in assigning keywords, the taxonomy satisfied our first criterion for validity and reliability.

Keyword frequency analysis Activity details Next, we selected a set of articles as a representative sample for the field of engineering education research in the United States, and we assigned and analyzed keywords for those articles. The set included 243 articles published during 2011, 2012, and 2013 in three journals: the Journal of Engineering Education, IEEE Transactions on Education, and the International Journal of Engineering Education. Although the number of articles published in these journals varied significantly, we analyzed the same number of articles from each journal to provide equal representation. Specifically, because the Journal of Engineering Education published the fewest number of articles each year, we included every article published in that journal but chose a random sample of articles in the other two journals each year. In total, of the 680 articles published in these three journals in 2011, 2012, and 2013, we analyzed 243 journal articles (see Table 4).

For each abstract of the 243 journal articles, the same two of us who completed the previous norming exercise used the working guidelines we had developed to select three to six keywords from version 0.6 of the draft taxonomy. (Although some of the articles had author-assigned keywords, we intentionally did not use them in our analysis, because the keywords were not drawn from a common set of terms and because not every article had them.) We conducted a frequency analysis of the keywords to study the distribution of the keywords across the taxonomy.

Outcome Altogether, from the 438 available terms of version 0.6 of the draft taxonomy (we chose not to use any of the 14 branch names as keywords), we successfully identified relevant keywords for all 243 articles. In total we assigned 1,176 keywords, distributed across all 14 taxonomy branches (Table 5). Thus, because the set of articles we selected to represent the field of engineering education research could be adequately described by terms in the taxonomy, the taxonomy satisfied our second criterion for validity and reliability.

Table 5 Distribution of Keywords Across the Taxonomy Branches

\begin{tabular}{lr}
\hline Taxonomy branch & References \\
\hline 1. Assessment & 106 \\
2. Design & 24 \\
3. Diversity & 53 \\
4. Educational level & 31 \\
5. Educational setting & 115 \\
6. Educational technology & 108 \\
7. Instruction & 88 \\
8. Outcomes & 59 \\
9. Professional practice & 172 \\
10. Recruitment and retention & 121 \\
11. Related fields & 16 \\
12. Research approach & 29 \\
13. Theoretical frameworks & 225 \\
14. Teams & 29 \\
\hline
\end{tabular}


For this set of articles and using version 0.6 of the taxonomy, Figure 2 provides a visual representation of the keyword frequency analysis, and Figure 3 displays the analysis as a word cloud. In our analysis, 168 of the taxonomy terms were never selected as keywords, and the remaining 270 terms were assigned as keywords an average of 4.4 times (maximum $=39$, median = 2). The three most frequently used taxonomy terms were "survey," "case study," and "undergraduate." The keywords for the abstracts were distributed in a fairly even way, so the taxonomy satisfied our third criterion for validity and reliability.

Because we had involved the broader community in refining the taxonomy at several workshops, and because the purpose of the keyword frequency analysis was to validate the taxonomy, we chose not to make significant adjustments based on our frequency analysis. However, the taxonomy terms that were not chosen as keywords for this set of journal articles will serve as candidates for removal as the taxonomy is further refined through actual use.

2014 American Society of Engineering Education Annual Conference Activity details Finally, we applied version 0.6 of the draft taxonomy to papers accepted for presentation at the 2014 ASEE Annual Conference by the Educational Research and Methods Division (ERM) of ASEE, and we asked authors to confirm whether keywords we assigned were appropriate to represent their papers. We restricted our analysis to papers published by the ERM Division for three reasons. First, the taxonomy focused on engineering education

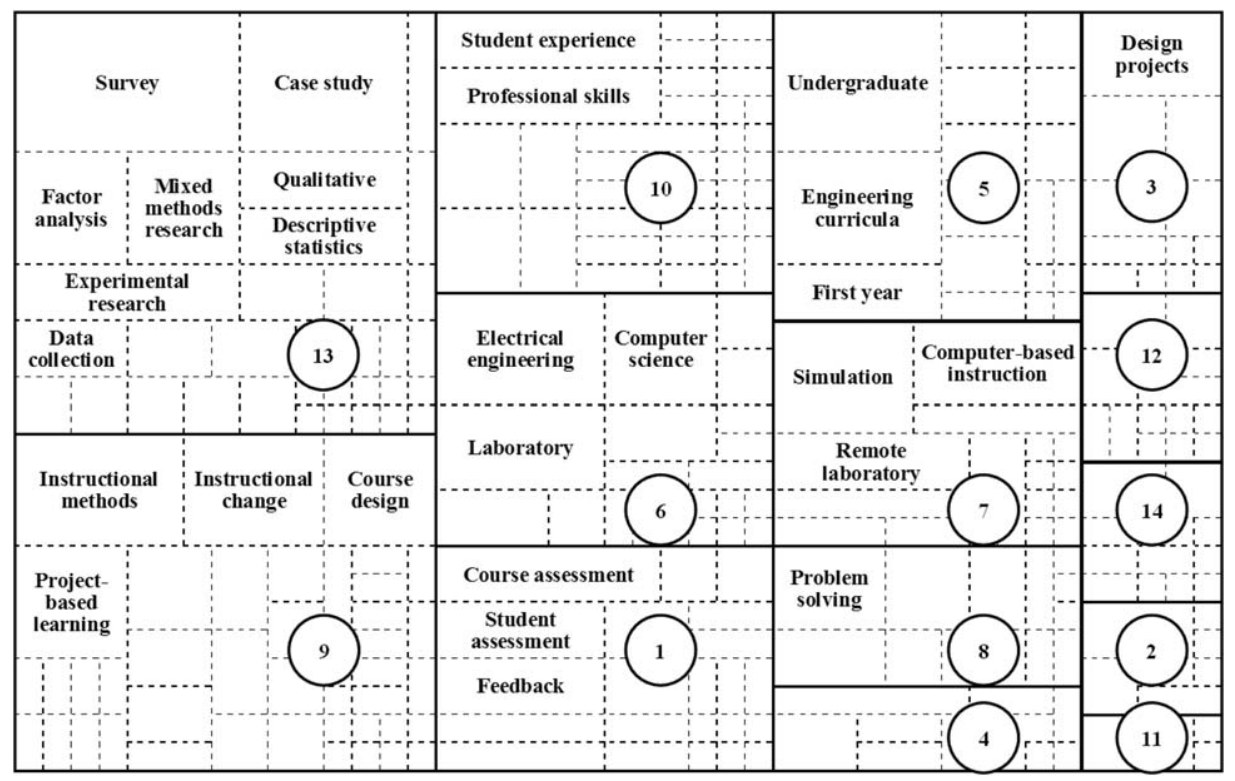

Figure 2 Keyword frequency analysis. The 270 taxonomy terms that are used at least once in the frequency analysis are each represented by an individual rectangular box, and the size of the box is proportional to the number of times a term was used. Terms that are used at least 10 times are listed. The dark borders separate terms in the 14 taxonomy branches, and those branches are identified by circled numbers. 


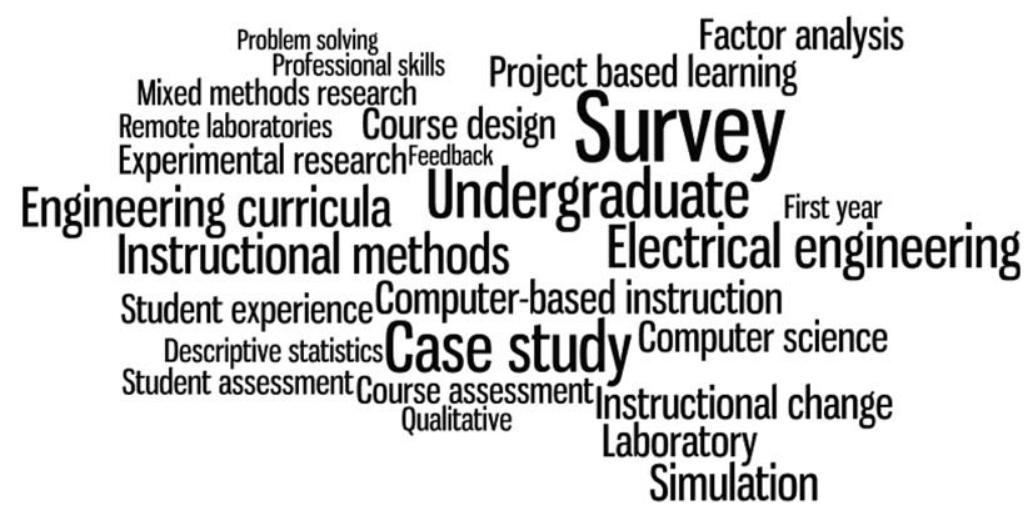

Figure 3 Word cloud for keyword frequency analysis. The 26 terms used at least 10 times in the keyword frequency analysis are illustrated here, and terms with larger font sizes were used more often.

research, and the ERM Division aligns most explicitly with this area. Second, limiting our analysis to these papers provided a manageable sample size. Third, we suspected that ERM members would be more likely to respond to requests related to taxonomy development than randomly selected authors.

In advance of the conference, we invited authors of all 70 ERM Division papers listed in the conference program to send the final version of their paper that would appear in the Proceedings of the 2014 ASEE Annual Conference. We received 36 papers, and we assigned five keywords (using the working guidelines we had established) to each. Next, we forwarded our set of keywords, along with our working guidelines for assigning them, to authors and requested feedback from the authors about how well they believed each keyword suited their paper. Authors were invited to offer alternative keywords if they disagreed with our selection. Thirty-one of the authors replied to our request.

Outcome The authors' level of agreement with the 155 keywords we assigned (five for each of the 31 papers) was quite high. Authors agreed or strongly agreed with 140 (90\%) of the keywords we selected and disagreed or strongly disagreed with 15 (10\%). This high author agreement suggested that the taxonomy will be usable in the future for authors to assign their own keywords when submitting abstracts and papers for peer review. Further, the strong agreement suggested that our approach was reliable and that the taxonomy satisfied our fourth criterion for validity and reliability.

In summary, our analyses showed that version 0.6 of the draft taxonomy satisfied all four of the criteria we established for validity and reliability. Multiple researchers selected reproducible keywords for a set of abstracts (Criterion 1), the taxonomy comprised terms that could describe every article in a representative sample from the field of engineering education research in the United States (Criterion 2), keywords selected for the sample were evenly distributed across the branches of the taxonomy (Criterion 3), and the strong agreement by authors with our researcher-assigned keywords indicated the reliability of keywords (Criterion 4). Following the validity and reliability testing, we made minor updates to version 0.6 of the draft taxonomy by removing duplicate terms, combining similar terms, renaming terms, 
and adding terms. The resulting version of the taxonomy, EER Taxonomy Version 1.0 (Finelli \& Borrego, 2015), has 454 terms arranged in 14 branches and six levels. It is our final one.

\section{Developing Guidelines for Assigning Keywords}

As we proposed previously, one primary use of this taxonomy for engineering education research is for selecting keywords that describe conference papers and journal articles. Providing keywords allows a researcher to describe scholarly work to a broader community, positions the work in the existing field, interests potential readers, and aligns current work with other useful research. Modern taxonomies often provide application guidelines for assigning keywords that address both content-related items, such as the type of keywords to assign (Levy, 2004), as well as broader guidelines, such as the total number of keywords to assign (Hedden, 2010).

During our interactions with multiple stakeholders in the development of the draft taxonomy, we identified multiple reasons for providing clear direction on assigning keywords. We found that, without guidance, authors often choose keywords that lack a uniform level of detail - the words are overly general or too specific. Similarly, authors use jargon or other terms that are not familiar to a broad range of researcher (rather than choosing preferred terms), and they use terms that sometimes have different meanings in different research contexts. We found that authors often select keywords that describe only a limited part of a project (for example, the population and intervention but not the methods or evidence), and they have difficulty gauging how many terms to apply.

Thus, concurrent with activities to develop and refine the taxonomy and assess its validity and reliability, we developed a set of guidelines to assist authors in assigning keywords to their publications. We reviewed author instructions for five journals targeted to engineering education research (Australasian Journal of Engineering Education, European Journal for Engineering Education, Journal of Engineering Education, IEEE Transactions on Education, and International Journal of Engineering Education) and summarized guidelines they provide for assigning keywords (Table 6). Then we drafted an initial set of guidelines that suggested that one or two keywords be assigned from each of three keyword categories: context/focus/topic, purpose/target/motivation, and research approach.

2014 Frontiers in Education Annual Conference Workshop details We solicited feedback about our initial guidelines during a free workshop at the 2014 Frontiers in Education (FIE) Annual Conference in Madrid, Spain, on October 22, 2014 (Finelli \& Rasoulifar, 2014). The abstract of the workshop printed in the conference program included an open invitation for all conference participants to attend. During the three-hour, pre-conference workshop, participants used the final taxonomy to choose keywords for four abstracts selected from papers published in the Proceedings of the 2011 FIE Annual Conference and in the Proceedings of the 2012 FIE Annual Conference, and they reflected on the approach they used for assigning keywords. Then, we introduced our initial set of guidelines, and participants repeated the keyword exercise; this time they compared keywords from the two activities and suggested improvements to the guidelines. Finally, workshop participants discussed, in small groups, ways to encourage other researchers and community members to use the taxonomy and ways to make it a more useful tool.

Outcome Participants found the guidelines helpful and easy to use, and they suggested updates that we incorporated into a final set of guidelines. The final guidelines are defined in Table 7 and mapped to related branches of the taxonomy from which keywords might be 
Table 6 Summary of Journal Instructions for Authors

Australasian Journal of Engineering Education

www.editorialmanager.com/eatj/default.aspx

Authors are instructed that each submission should include keywords, but no guidance is offered.

European Journal of Engineering Education

www.tandfonline.com/action/authorSubmission?journalCode $=$ ceee20\&page $=$ instructions\&\#.VJB3zCvF9PO

Instructions for authors note that "each manuscript should have 3 to 6 keywords," and they provide hyperlinks to generic guidelines that encourage authors to refer to an "established common indexing standard in a particular discipline (e.g., GeoRef, ERIC Thesaurus, PsycInfo, ChemWeb, and so on).” However, specific advice about keywords is not given.

Journal of Engineering Education

onlinelibrary.wiley.com/journal/10.1002/\%28ISSN\%292168-9830/homepage/ForAuthors.html

When submitting the structured abstract that accompanies each manuscript, authors are instructed to "list three keywords," but no further guidance is provided.

IEEE Transactions on Education

www.ieee.org/publications_standards/publications/authors/authors_journals.html

The journal provides a taxonomy of suggested keywords and encourages authors to select terms from the list (or to add their own terms if they cannot find appropriate ones). The list comprises "the first three hierarchical levels under each branch that is formed from the top-most terms of the IEEE Thesaurus." The 2014 IEEE Taxonomy, however, frequently does not include education-related terms (e.g., it does not include the terms "gender," "pedagogy," "focus group," or "design project"), so it is only of limited use. There are no other guidelines for assigning keywords.

International Journal of Engineering Education www.ijee.ie/authors/Guide_to_Authors.html

Instructions for assigning keywords are limited to "include keywords such as: keyword1; keyword2; keyword3; keyword4."

chosen. Table 8 gives an example of how the guidelines can be applied to assign keywords for an abstract.

To encourage more widespread use of the taxonomy, workshop participants suggested that (1) journal editors and conferences should begin requiring its use, (2) the taxonomy should be used to index conference proceedings and other collections and to cluster papers according to related keywords, and (3) a marketing campaign should be undertaken to illustrate the utility of the taxonomy. Finally, on the basis of their interactions with different electronic and paper versions of the taxonomy, participants suggested the taxonomy should be packaged as a free iPhone app or a mobile website to make it a more useful tool. We have responded by developing both tools.

In summary, using these guidelines can ensure that the keywords are assigned to identify important concepts and are chosen in a consistent way. And although they were developed to assist authors in assigning keywords to their publications, the guidelines might also assist reviewers in describing their own research expertise.

\section{The Final Taxonomy}

The final taxonomy, EER Taxonomy Version 1.0 (Finelli \& Borrego, 2015), comprises 455 terms arranged in 14 branches and six levels. It is copyrighted under a Creative Commons 
Table 7 Guidelines for Selecting Keywords

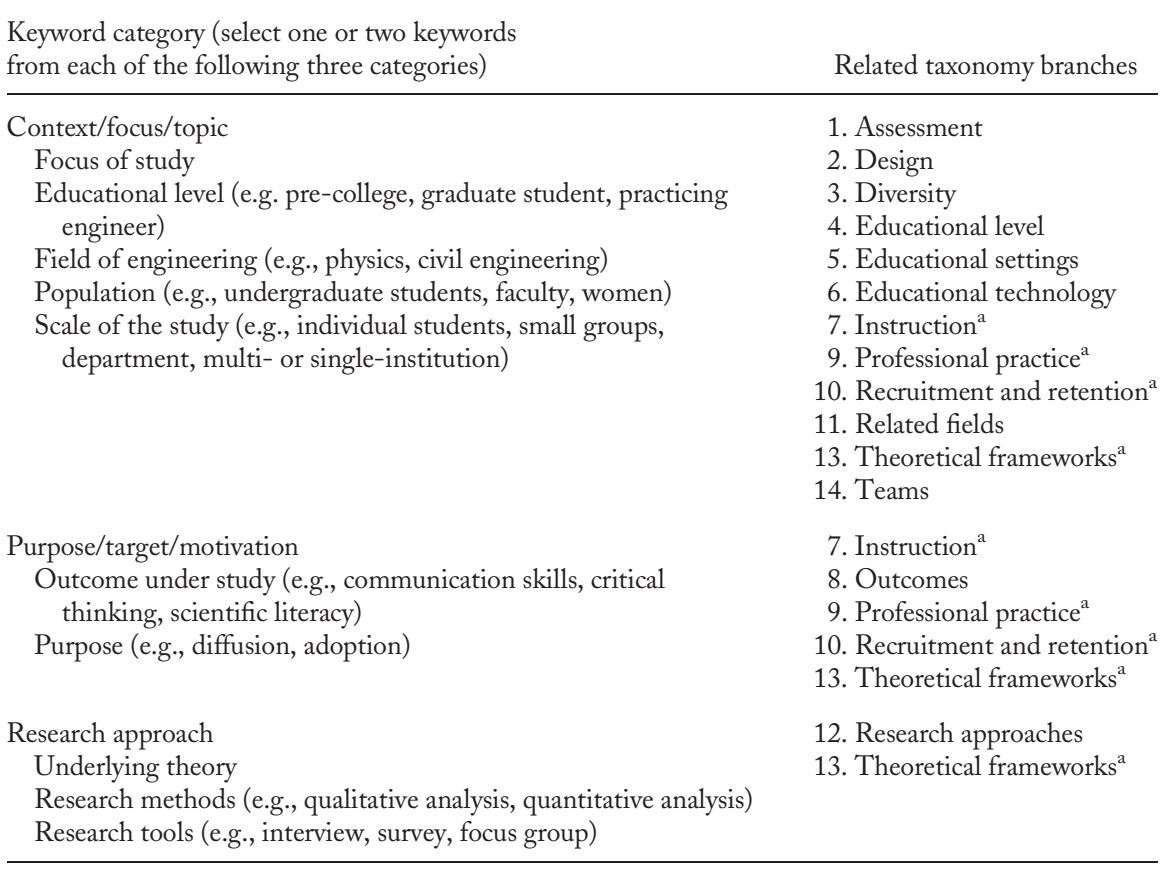

${ }^{a}$ Words appearing in these taxonomy branches are relevant for multiple keyword catagories.

Attribution-Share Alike 4.0 International License and is available to use and adapt for free with attribution to the original source. This final version was developed in concert with a professional taxonomist, and the full taxonomy is archived and is accessible from the institutional repositories for the first author's current institution (the University of Michigan at Ann Arbor).

An iPhone app, EER Taxonomy (Finelli, 2015), is built for iOS 8 and provides an easyto-use interface for the taxonomy. The application automatically updates the taxonomy from a remote server (obviating the need for a user to download updates), and it includes a brief user's guide. The app provides functionality to interactively browse the taxonomy in a multilevel environment to reflect the outline structure of the taxonomy. As well, users can conduct an instant search, and the location of the resulting keyword is indicated through a combination of breadcrumb navigation and expandable terms. Users may create, edit, delete, and export customized lists of favorite or commonly used keywords. The app, which allows users to send feedback to the research team and which links to a Creative Commons license, may be downloaded for free from the Apple app store. Users will also be able to access updates of the taxonomy from the website (http://taxonomy.engin.umich.edu).

A password-protected Internet application provides administrator access for the taxonomy. It offers an automated way to update the taxonomy by importing a spreadsheet of taxonomy terms or by adding or removing words directly. The administrator app incorporates web security best practices, allows for user-management, includes password protection and recovery, and allows the administrator to generate a printable version (from a PDF) of the taxonomy for distribution. 
Table 8 Example Application

of Keyword Guidelines

Sample abstract (Finelli et al., 2014)

Student resistance is often cited as a major barrier to faculty's use of active learning, but there are few researchbased strategies for reducing this barrier. To address the need for such strategies, we have initiated a project to identify specific, research-based strategies to significantly reduce student resistance to faculty's use of active learning practices. In this work-in-progress paper, we describe the first phase of our research - the development and pilot testing of a classroom observation instrument to assess student responses to faculty's use of active learning. This instrument, which draws upon other published observation protocols, will allow us to capture data about faculty's use of and students' response to active learning as we undertake our larger research project.

Selected keywords

From context/focus/topic category:

From purpose/target/motivation category:

From research approach category:

active learning, student perception

instructional change

classroom, observations

\section{Discussion}

This project had two major goals: create a useful taxonomy and establish an inclusive process. We succeeded in achieving both.

The inclusive approach we used in developing the taxonomy helped produce a useful product. We purposefully engaged the community at multiple, iterative stages of development; this process produced a taxonomy that will be broadly accessible by members of the community and that will allow researchers to see how their work can be represented using its terms. We developed a series of activities for individuals to interact with and navigate the taxonomy, and we focused the activities on abstracts of recently published papers that were representative of the conference that participants were attending. Because participants reviewed abstracts (rather than full papers), these activities highlighted characteristics of well-written abstracts and, by doing so, had a secondary benefit of educating individuals about how to write better abstracts.

Because users of a taxonomy should share a common understanding of how to apply it, we developed and provided guidelines for assigning keywords. The guidelines help overcome the common mistakes of assigning keywords that are overly specific, that are unlikely to be used by others, or that are too general to sufficiently communicate the unique characteristics of the work. Selecting keywords in a consistent way can increase the number of readers who locate a scholarly work and can facilitate better connections between related activities. Other aspects of a useful taxonomy include uniform depth and specificity (Pack, 2002), and we ensured that ours did through our careful revisions that incorporated community feedback, advice from an advisory board, and insights from a professional taxonomist.

We established an inclusive process in the development of the taxonomy in several ways. Rather than using invitation-only activities that would exclude some members of the community, we issued open calls for participation that we distributed widely. We used transparent procedures to select participants when space was limited, and our selection criteria emphasized diversity of perspectives. Through a variety of evolving activities at conferences around the world, we engaged a large number of participants at various career stages. We also learned more inclusive ways to develop the taxonomy. We found from the Mapping the Field Conference that some participants inferred that the outline structure of the taxonomy implied a hierarchical ordering of the terms. Many groups offered suggestions for new branches, and two groups chose to reorganize the entire list of terms. Participants argued that specific topics 
were foundational and deserved to be main headings in the hierarchy. We reflected on our experiences at that conference; then we altered our subsequent activities to emphasize that the outline was merely intended as an organizational structure to provide context, to further define the terms, and to help authors and indexers find terms. We also systematically arranged taxonomy terms in alphabetical order within the outline to make these points even clearer. Further, we decided early in our work that prioritizing important research areas would be detrimental to our goal of creating a comprehensive map for the field, so we purposefully decoupled discussions about a research agenda from development of a complete taxonomy.

\section{Summary and Recommendations}

Although taxonomies are in widespread use, there is little empirically based literature to guide development and validation of new ones. We enlisted expertise from a professional taxonomist and the engineering education research community to adapt known procedures to develop a useful taxonomy through an inclusive process. Once our taxonomy is in place and authors routinely assign keywords to their publications, the field will be in a better position to make evidence-based decisions about gaps and priorities for future research.

We offer advice for others undertaking similar initiatives. Authors know their work best and therefore should assign their own keywords. Linking abstract and paper submission portals to a taxonomy empowers authors to index their own work and helps generate the types of metadata that will be useful for analyzing publication trends in the field. Users, though, need guidelines for assigning keywords to help balance specificity with the need to use a common set of terms to connect related work. Throughout this project, we spent significant time developing and testing guidelines with potential users. These guidelines increased reliability, and users indicated satisfaction with them. Since different journals represent different perspectives on the field and different regional foci or interests, it was important to consider publications from multiple sources in developing, refining, and validating the taxonomy.

\section{Future Work}

One limitation of this project is that, due to time and funding constraints, the taxonomy focuses on engineering education research in the United States. Although our process was inclusive of some members of the international engineering education research community, and we received some feedback about ways to reflect different approaches and terminologies used outside of the United States, we did not aim to develop a truly international taxonomy. Among other things, doing so would involve using a different set of preferred terms, mapping preferred terms for the United States to those preferred in other regions, and introducing different or expanded branches to reflect other research emphases. Expanding our current taxonomy or recreating the process on a more international scale is one potential direction for future work.

Future work also involves making ongoing updates and refinements to the current taxonomy. Although the organization of the terms within the taxonomy provides contextual cues for the definition of each term, participants frequently requested definitions for all terms. Writing and vetting definitions, providing examples of the keywords in practice, and fully cross-referencing related terms are all future work that would help reduce confusion and ambiguity for users. In addition, for the taxonomy to remain relevant and usable, the list of terms needs to be updated regularly (Levy, 2004). In other fields, the approach for regularly 
maintaining a taxonomy consists of having one or a few taxonomists who accept and evaluate suggestions for new terms, monitor the taxonomy for outdated terms that can be deleted or merged with others, and monitor the use of the terms and provide clarification when needed (Hedden, 2010). On a longer but still regular schedule, a broader cross section of the community would be involved in updating the taxonomy, for example every five or 10 years.

We propose our field adopt similar mechanisms to allow community members to suggest new terms for addition to the taxonomy by establishing a committee of representatives from the journals jointly publishing this report. The committee could manage the periodic evaluation of community suggestions to ensure that the taxonomy remains relevant and useful to the engineering education research community in the future.

Consistent with our inclusive approach, we synthesized the suggestions of hundreds of community members to produce our final version of the taxonomy. Future work will include procedures for adding new terms as they are needed. The taxonomy itself is copyrighted under a Creative Commons license, which allows others to adapt and update the work with attribution to the original. We hope the Creative Commons licensing promotes continued community ownership and updates of the taxonomy so that it remains relevant and useful.

Finally, many of the features of the iPhone app are consistent with expert recommendations for displaying taxonomies (Hedden, 2010). We will continue to solicit suggestions for updating the app. Future features of the app will include a way to suggest new terms for the taxonomy and a more seamless interface with manuscript submission sites so authors can use the app to select keywords that are automatically uploaded to the journal's manuscript submission site.

\section{Acknowledgments}

This material is based upon work supported by the National Science Foundation under EEC Grant 1240797. Any opinions, findings, and conclusions or recommendations expressed are those of the authors and do not necessarily reflect the views of the National Science Foundation. The authors wish thank members of the advisory board (listed in Table 3) for offering guidance throughout the project, Marjorie Hlava and her Access Innovations colleagues for providing good insight as we refined the taxonomy, Samuel Scott for creating the iPhone app, Charlotte Sawyer for assisting with validating the taxonomy, and Robyn Rosenberg for helping identify relevant literature.

\section{References}

Alpay, E., \& Jones, M. E. (2012). Engineering education in research-intensive universities. European Journal of Engineering Education, 37(6), 609-626.

Besterfield-Sacre, M., Cox, M. F., Borrego, M., Beddoes, K., \& Zhu, J. (2014). Changing engineering education: Views of U.S. faculty, chairs, and deans. Journal of Engineering Education, 103(2), 193-219.

Borrego, M. (2007). Development of engineering education as a rigorous discipline: A study of the publication patterns of four coalitions. Journal of Engineering Education, 96(1), 5-18.

Borrego, M. (2013). Refining a taxonomy for engineering education research. Workshop at the 2013 European Society for Engineering Education (SEFI) Annual Conference, Leuven, Belgium. Retrieved from http://www.kuleuven.be/communicatie/congresbureau/oud2015/ congres/sefi2013/programme\#Friday 20th/ 
Borrego, M., \& Bernhard, J. (2011). The emergence of engineering education research as an internationally connected field of inquiry. Journal of Engineering Education, 100(1), 14-47.

Case, J. M., \& Light, G. (2011). Emerging research methodologies in engineering education research. Journal of Engineering Education, 100(1), 186-210.

de Graaff, E., \& Kolmos, A. (2014). Innovation and research on engineering education. In Handbook of research on educational communications and technology (pp. 565-571). New York, NY: Springer.

Finelli, C. J. (2013). Refining a taxonomy for engineering education research. Workshop at the 2013 Frontiers in Education Annual Conference, Oklahoma City, OK. doi: 10.1109/ FIE.2013.6684779

Finelli, C. J. (2015). EER Taxonomy (Version 1.0). [Mobile application software]. Retrieved from https://itunes.apple.com/us/app/eer-taxonomy/id987326779? $\mathrm{mt}=8$

Finelli, C. J., \& Borrego, M. (2013). Refining a taxonomy for engineering education research. Workshop at the 2013 ASEE Annual Conference, Atlanta, GA. Retrieved from http:// www.asee.org/public/conferences/20/registration/view_session?session_id=2366

Finelli, C. J., \& Borrego, M. (2014). An inclusive process for developing a taxonomy of keywords for engineering education research. Proceedings of the 2014 ASEE Annual Conference, Indianapolis, IN. Retrieved from https://peer.asee.org/20057

Finelli., C., \& Borrego, M. (2015). EER Taxonomy Version 1.0. Retrieved from University of Michigan website: http://taxonomy.engin.umich.edu/wp-content/uploads/2015/08/ EER-Taxonomy-Version-1.0.docx

Finelli, C. J., DeMonbrun, M., Borrego, M., Shekhar, P., Henderson, C., Prince, M. J., \& Waters, C. K. (2014). A classroom observation instrument for assessing student reaction to active learning. Proceedings of the 2014 Frontiers in Education Annual Conference, Madrid, Spain. doi: 10.1109/FIE.2014.7044084

Finelli, C. J., \& Rasoulifar, G. (2014). Using and disseminating a taxonomy for engineering education research. Workshop at the 2014 Frontiers in Education Annual Conference, Madrid, Spain. doi: 10.1109/FIE.2014.7043989

Froyd, J. E., Wankat, P. C., \& Smith, K. A. (2012). Five major shifts in 100 years of engineering education. Proceedings of the IEEE, 100, 1344-1360.

Hawkins, D. T., Larson, S. E., \& Caton, B. Q. (2003). Information science abstracts: Tracking the literature of information science. Part 2: A new taxonomy for information science. Journal of the American Society for Information Science E Technology, 54(8), 771-781.

Hedden, H. (2010). The accidental taxonomist. Medford, NJ: Information Today.

Heywood, J. (2005). Engineering education: Research and development in curriculum and instruction. Hoboken, NJ: John Wiley \& Sons.

Jesiek, B. K., Borrego, M., Beddoes, K., Hurtado, M., Rajendran, P., \& Sangam, D. (2011). Mapping global trends in engineering education research, 2005-2008. International Journal of Engineering Education, 27(1), 77-90.

Jesiek, B. K., Newswander, L. K., \& Borrego, M. (2009). Engineering education research: Discipline, community, or field? Journal of Engineering Education, 98(1), 39-52.

Kalmukov, Y. (2012). Describing papers and reviewers' competences by taxonomy of keywords. Computer Science and Information Systems, 9(2), 764-789.

Kelly, E. (2014). Design-based research in engineering education: Current steps and next steps. In A. Johri \& B. Olds (Eds.), Cambridge handbook of engineering education research (pp. 497-517). New York, NY: Cambridge University Press. 
Levy, T. (2004). Standard taxonomies offer flexibility. Seybold Report: Analyzing Publishing Technologies, 4(8), 9-11.

Liu, Q., Vorvoreanu, M., Madhavan, K., \& McKenna, A. F. (2013). Designing discovery experience for big data interaction: A case of web-based knowledge mining and interactive visualization platform. Proceedings of the International Conference on Human-Computer Interaction, Las Vegas, NV. doi:10.1007/978-3-642-39253-5_60

Malmi, L., Adawi, T., Curmi, R., de Graaff, E., Duffy, G., Kautz, C., . . . Williams, B. (2013). Methodological analysis of SEFI EER papers. Proceedings of the 2013 European Society for Engineering Education (SEFI) Annual Conference, Leuven, Belgium. Retrieved from http://www.sefi.be/conference-2013/images/87.pdf

Malmi, L., de Graaff, E., Adawi, T., Curmi, R., Duffy, G., Kautz, C., . . . Williams, B. (2012). Developing a methodological taxonomy of EER papers. Proceedings of the 2012 European Society for Engineering Education (SEFI) Annual Conference, Thessaloniki, Greece. Retrieved from http://www.sefi.be/conference-2012/Papers/Papers/069.pdf

Mayor, C., \& Robinson, L. (2014). Ontological realism, concepts and classification in molecular biology. Journal of Documentation, 70(1), 173-193.

Pack, T. (2002). Taxonomy's role in content management. EContent, 25(3), 26-31.

Ulrich, W., \& Dash, D. P. (2011). Introducing a concept hierarchy for the Journal of Research Practice. Journal of Research Practice, 7(2), 1-21.

Wankat, P. C. (1999). An analysis of the articles in the Journal of Engineering Education. Journal of Engineering Education, 88(1), 37-42.

Wankat, P. C. (2004). Analysis of the first ten years of the Journal of Engineering Education. Journal of Engineering Education, 93(1), 13-21.

Whitin, K., \& Sheppard, S. (2004). Taking stock: An analysis of the publishing record as represented by the Journal of Engineering Education. Journal of Engineering Education, 93(1), 5-12.

Xian, H., \& Madhavan, K. (2014). Anatomy of scholarly collaboration in engineering education: A big-data bibliometric analysis. Journal of Engineering Education, 103(3), 486-514.

\section{Authors}

Cynthia J. Finelli is an associate professor of electrical engineering and computer science and the director of the Center for Research on Learning and Teaching in Engineering at University of Michigan, 208 Gorguze Family Laboratory, 2609 Draper Drive, Ann Arbor, MI 48109-2101; cfinelli@umich.edu.

Maura Borrego is an associate professor in mechanical engineering and in curriculum and instruction at the University of Texas at Austin, 204 E. Dean Keeton Street, Stop C2200, Austin, TX 78712-1591; maura.borrego@austin.utexas.edu.

Golnoosh Rasoulifar served as a postdoctoral research fellow at the Center for Research on Learning and Teaching in Engineering at University of Michigan; golnoosh.rasoulifar@ gmail.com. 